\title{
A comparative study of hyperelastic constitutive models to characterize the behavior of a polymer used in automotive engines
}

\author{
Rafael Tobajas *, Elena Ibarz and Luis Gracia \\ University of Zaragoza, C/María de Luna 350180 Zaragoza, Aragón, Spain E-mails: \\ rafaeltobajasalonso@gmail.com (R.T.); eibarz@unizar.es (E.I.); lugravi@unizar.es (L.G.) \\ * Correspondence: rafaeltobajasalonso@gmail.com (R.T.); Tel.: +34 876555211
}

Received: 9 April 2016; Accepted: 26 April 2016; Published: 2 May 2016

\begin{abstract}
The global automotive industry faces the challenge of increasing engine efficiency, reducing fuel consumption and the size of them gradually. Not only the engine block must reduce its size, but also other components, requiring more compact and flexible designs using materials such as thermoplastic elastomers. That kind of materials are used due to their characteristics, such as ability of deformation, durability, recyclability, and its cost/weight ratio. They are able to hold large deformations and they have very good damping characteristics, making them suitable for use in energy dissipation. Characterization of the dynamic mechanical properties of these materials is essential to make a correct analysis and modeling of the behavior of components. Although the constitutive models of these materials are complex due to high deformability, quasi-incompressibility, softening, and time dependent effects, typically, these materials have a mechanical behavior which can be represented by a phenomenological hyperelastic model. While it is easy to fit a model of elastic behavior, set a model for a hyperelastic material is a very complex task, so in practice simplified models are used. This paper proposes a comprehensive comparison of six hyperelastic models to simulate the behavior of Santoprene 101-73 material manufactured by ExxonMobil. The ability of these models to reproduce different types of loading conditions is analyzed through uniaxial tensile data obtained experimentally. The parameters of each of the hyperelastic models are determined by a least-squares fit and then a classification of these six models is established, highlighting those that are most suitable for characterizing the material.
\end{abstract}

Keywords: Thermoplastic elastomers; hyperelastic; Santoprene.

\section{Introduction}

During the last decades the use of polymeric materials has been increased significantly in key industry sectors such as the automotive sector [1,2]. This is mainly due to the technological capacity of these materials to achieve complex geometries with assumable economic and time costs [3]. In addition, they have other advantages over other materials such as its low density and its thermal and electrical insulation [4].

In the automotive industry, within the family of polymers, thermoplastic elastomeric materials have an increasingly important role [5]. Besides they are used as the main material in such important components as tires, they play an essential role in components such as air ducts engine [6]. Lightness, manufacturing capacity, deformability and vibration absorption capability [4] of these materials make them suitable for the manufacture of these elements allowing to produce increasingly compact, lightweight and efficient engines that reduce fuel consumption.

Despite its advantages, the design and mechanical analysis using these materials is not a simple task on account of they have a non-linear and time dependent mechanical behavior. In adittion, they undergo softening and they are quasi-incompressible [7]. This makes that simulations with linear 
elastic models have not precise agreement with the real behavior and unsatisfactory results are obtained. Those inaccurate results do not guarantee the correct functionality of a particular design.

This paper presents a search for a hyperelastic model that can reproduce as accurately as possible the mechanical behavior of a material of this type. The chosen material is Santoprene 101-73 manufactured by the ExxonMobil [8] and used to make air intake tubes in automotive engines.

The main objective is to simulate the mechanical behavior of the material, theoretically and practically, under large deformations. To perform this, a study of six widely referenced hyperelastic models in the literature is performed:

- Neo Hookean

- Mooney-Rivlin

- Ogden

- Gent-Thomas

- Arruda-Boyce

- Yeoh

From the formulation of strain energy density function of each of models, an analytical development for calculating stresses in the uniaxial case will be made. The parameters of each of models will be obtained by a least squares fit algortihm. The results will be compared with the test provided by the manufacturer. The correlation coefficient $\mathrm{R}^{2}$ will be used to know which model best represents the actual mechanical behavior of the material.

\section{Material}

The nonlinear behavior of the material has been described by uniaxial tensile tests on sample parts produced by the manufacturer. The datasheet of material describes the mechanical behavior for axial tension, biaxial tension and compression. Furthermore, information on the cyclic behavior of the material is also provided at different levels of deformation.

From the information on the cyclical behavior of the material, the material behavior to the first pull deformation can be obtained. This behavior is shown by a solid red line in Figure 1 .

In order to obtain values of strain energy density, stress values from the first pull deformation has been obtained. Strain energy density values were obtained by Equation (1) and are shown in Table 1.

$$
W=\sum_{i, j=1}^{3} \int_{0}^{\varepsilon_{i j}} \sigma_{i j} d \varepsilon_{i j}
$$




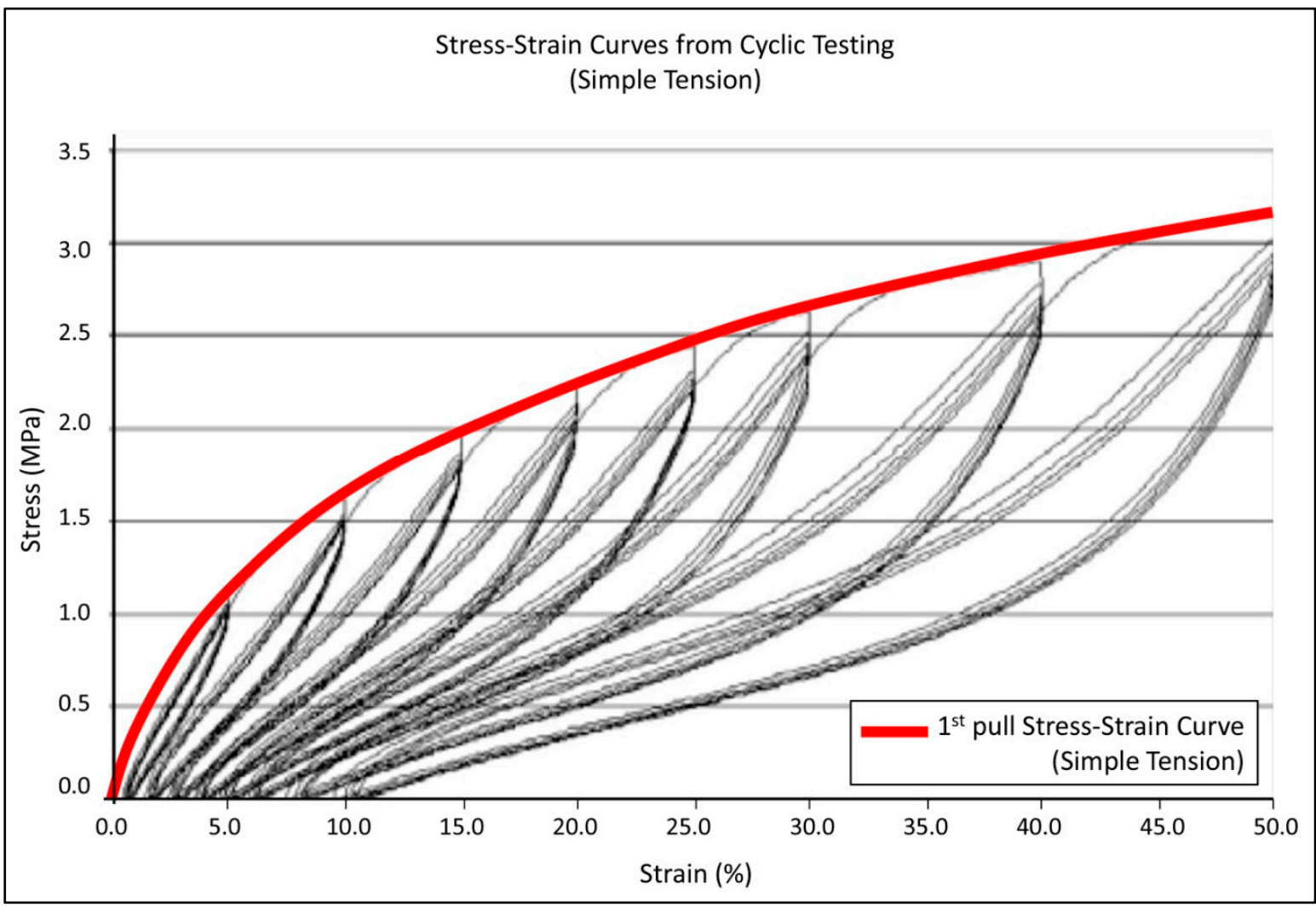

Figure 1. Strain-stress plot of Santoprene 101-73 from manufacturer datasheet [8].

Table 1. Strain, stress, strain energy density data of Santoprene 101-73 from manufacturer datasheet.

\begin{tabular}{ccc}
\hline Strain (\%) & Stress (Mpa) & W(SED) \\
\hline 0,000 & 0,000 & 0,000 \\
\hline 0,935 & 0,345 & 0,002 \\
\hline 1,992 & 0,615 & 0,006 \\
\hline 3,240 & 0,859 & 0,015 \\
\hline 4,657 & 1,084 & 0,029 \\
\hline 6,868 & 1,338 & 0,056 \\
\hline 8,704 & 1,516 & 0,082 \\
\hline 9,896 & 1,623 & 0,101 \\
\hline 12,187 & 1,792 & 0,140 \\
\hline 13,778 & 1,913 & 0,170 \\
\hline 14,950 & 1,981 & 0,192 \\
\hline 17,629 & 2,122 & 0,246 \\
\hline 18,941 & 2,193 & 0,274 \\
\hline 19,903 & 2,232 & 0,296 \\
\hline
\end{tabular}

\begin{tabular}{ccc} 
Strain (\%) & Stress (Mpa) & W(SED) \\
\hline 22,987 & 2,374 & 0,366 \\
\hline 24,211 & 2,422 & 0,396 \\
\hline 24,963 & 2,451 & 0,414 \\
\hline 28,425 & 2,585 & 0,501 \\
\hline 29,876 & 2,631 & 0,539 \\
\hline 33,938 & 2,768 & 0,648 \\
\hline 35,879 & 2,819 & 0,702 \\
\hline 37,772 & 2,869 & 0,756 \\
\hline 39,748 & 2,905 & 0,813 \\
\hline 45,436 & 3,079 & 0,982 \\
\hline 47,482 & 3,112 & 1,046 \\
\hline 48,864 & 3,136 & 1,089 \\
\hline 49,860 & 3,149 & 1,120
\end{tabular}

\section{Basics of continuum mechanics}

Normally, hyperelastic models are characterized by strain energy function density $\mathrm{W}$ which is formulated as a function depending on different magnitudes associated to the strain field and the material constants. Usually: 


$$
W=W(F)=W\left(\lambda_{1}, \lambda_{2}, \lambda_{3}\right)=W\left(I_{1}, I_{2}, I_{3}\right)
$$

where $\lambda_{1}, \lambda_{2}, \lambda_{3}$ are principal stretches, and $\mathrm{I}_{1}, \mathrm{I}_{2}, \mathrm{I}_{3}$ are the invariants of left Cauchy-Green strain tensor, $\boldsymbol{B}$, respectively, obtained as:

$$
\begin{gathered}
I_{1}=\operatorname{trace}(B) \\
I_{2}=\frac{1}{2}\left(I_{1}^{2}-B \cdot B\right) \\
I_{3}=\operatorname{det}(B)
\end{gathered}
$$

Stress-strain relations are derived by differentiating the strain energy density function through the formulation described below.

If the strain energy function is defined in terms of strain invariants $I_{1}, I_{2}, I_{3}$ :

$$
\sigma_{i j}=\frac{2}{\sqrt{I_{3}}}\left[\left(\frac{\partial W}{\partial I_{1}}+I_{1} \frac{\partial W}{\partial I_{2}}\right) B_{i j}-\frac{\partial W}{\partial I_{2}} B_{i k} B_{k j}\right]+2 \sqrt{I_{3}} \frac{\partial W}{\partial I_{3}} \delta_{i j}
$$

If the strain energy function is defined in terms of the principal stretches $\lambda_{1}, \lambda_{2}, \lambda_{3}$ :

$$
\sigma_{i j}=\frac{\lambda_{1}}{\lambda_{1} \lambda_{2} \lambda_{3}} \frac{\partial W}{\partial \lambda_{1}} b_{i}^{(1)} b_{j}^{(1)}+\frac{\lambda_{1}}{\lambda_{1} \lambda_{2} \lambda_{3}} \frac{\partial W}{\partial \lambda_{2}} b_{i}^{(2)} b_{j}^{(2)}+\frac{\lambda_{1}}{\lambda_{1} \lambda_{2} \lambda_{3}} \frac{\partial W}{\partial \lambda_{3}} b_{i}^{(3)} b_{j}^{(3)}
$$

where $b_{1}, b_{2}$, $b_{3}$ are three orthogonal unit vectors which correspond to $B$ tensor eigenvectors and they define the three principal directions of deformation.

\section{Hyperelastic models}

To obtain a constitutive model that can reproduce with the maximum possible accuracy the behavior of the material, six classic hyperelastic models were analyzed. In this section the six models studied (and corresponding sub-models) and their formulation are described.

\subsection{Mooney-Rivlin model}

Mooney-Rivlin model $[9,10]$, proposed in 1951, is one of the hyperelastic models most used in the literature. Although there are various versions of this model, the most general are based on the first and second invariants deformation. The strain energy density function is defined as follows:

$$
W=C_{10}\left(I_{1}-3\right)+C_{01}\left(I_{2}-3\right)+\frac{1}{D}(J-1)^{2}
$$

where $\mathrm{C}_{\mathrm{ij}}$ are material constants, $\mathrm{J}$ is the determinant of the strain gradient tensor $\mathrm{F}$ and $\mathrm{D}$ is a material constant related to the bulk modulus.

\subsection{Neo-Hookean model}

This model was proposed by Treloar in 1943 [11], and it is a particular case of two parameters Mooney-Rivlin model. In this model, the strain energy density function is based only on the first strain invariant: 


$$
W=C_{1}\left(I_{1}-3\right)+\frac{1}{D}(J-1)^{2}
$$

where $C_{1}$ is a material constant, $J$ is the determinant of the strain gradient tensor $F$ and $D$ is a material constant related to the bulk modulus.

\subsection{Ogden model}

Ogden hyperelastic model (1972) [12] is possibly the most extended model after Mooney-Rivlin model. This model is based on the three principal stretches $\left(\lambda_{1}, \lambda_{2}, \lambda_{3}\right)$ and $2 \cdot \mathrm{N}$ material constants, where $\mathrm{N}$ is the number of polynomials that constitute the strain energy density function, defined as:

$$
W=\sum_{i=1}^{N} \frac{\mu_{i}}{\alpha_{i}}\left(\lambda_{1}^{\alpha_{i}}+\lambda_{2}^{\alpha_{i}}+\lambda_{3}^{\alpha_{i}}-3\right)+\sum_{k=1}^{N} \frac{1}{D}(J-1)^{2 k}
$$

where $\mu_{\mathrm{i}} \mathrm{y} \alpha_{\mathrm{i}}$ are material constants, $\mathrm{J}$ is the determinant of the strain gradient tensor $\mathrm{F}$ and $\mathrm{D}$ is a material constant related to the bulk modulus.

\subsection{Gent-Thomas model}

Gent-Thomas model [13] is a hyperelastic constitutive model that uses three material constants. It is based on the first and second strain invariants. The strain energy density function is defined as follows:

$$
W=C_{1}\left(I_{1}-3\right)+3 C_{2} \ln \left(I_{2}\right)+\frac{1}{2} \lambda\left[(\ln J)^{2}+(J-1)^{2}\right]
$$

where $C_{1}, C_{2}$ and $\lambda$ are material constants, and $J$ is the determinant of the strain gradient tensor $F$.

\subsection{Yeoh model}

The model proposed in 1990 by Yeoh [14], is based on the first strain invariant. The strain energy density function is defined as follows:

$$
W=\sum_{i=1}^{N} C_{i 0}\left(I_{1}-3\right)^{i}+\sum_{k=1}^{N} \frac{1}{D}(J-1)^{2 k}
$$

where $\mathrm{C}_{\mathrm{i} 0}$ are material constants, $\mathrm{J}$ is the determinant of the strain gradient tensor $\mathrm{F}$ and $\mathrm{D}$ is a material constant is related to the bulk modulus.

\subsection{Arruda-Boyce model}

The micromechanical model Arruda-Boyce [15] was proposed in 1993 and is a particular case of Yeoh model with $\mathrm{N}=5$. It is based on the first strain invariant and is also known as teightchain model. The strain energy density function is defined as follows:

$$
W=\mu \sum_{i=1}^{N} \frac{C_{i}}{\lambda_{L}^{2 i-2}}\left(I_{1}^{i}-3^{i}\right)+\frac{1}{D}\left[\frac{J^{2}-1}{2}-\operatorname{lnJ}\right]
$$

where $C_{i} y \lambda_{\mathrm{L}}$ are material constants, $\mu$ is the initial shear modulus , $\mathrm{J}$ is the determinant of the strain gradient tensor $\mathrm{F}$ and $\mathrm{D}$ is a material constant related to the bulk modulus.

\section{Model fitting}

All models presented in the previous section are dependent on strain magnitudes and material constants. To obtain the optimum values of these constants that make each of the models reproduce faithfully the data from manufacturer, an optimization algorithm was used. The discrepancy 
between theoretical and real data has been defined as the error of the model. A least squares algorithm was used to fit the values of material constants by minimizing the error. The error function is defined as:

$$
\phi=\text { Error }_{\text {model }}=\sum_{i=1}^{N}\left(\text { Data }_{\text {real }}-D a t a_{\text {model }}\right)^{2}
$$

where $\mathrm{N}$ is the number of points on the chart provided by the manufacturer.

To determine the quality of each of the models, the $\mathrm{R}^{2}$ correlation coefficient was calculated.

As a result of the models, a strain energy density curve and a stress-strain curve were obtained. This stress-strain curve will be compared with the actual material curve. The best hyperelastic model will be the one whose average values $\mathrm{R}^{2}$ (strain energy density and stress) is closer to 1 .

\section{Results}

In this section, the results obtained for each of the models (and sub-models) are presented. The strain energy density function curves and stress-strain curves are shown in Fig. 2 to 7.

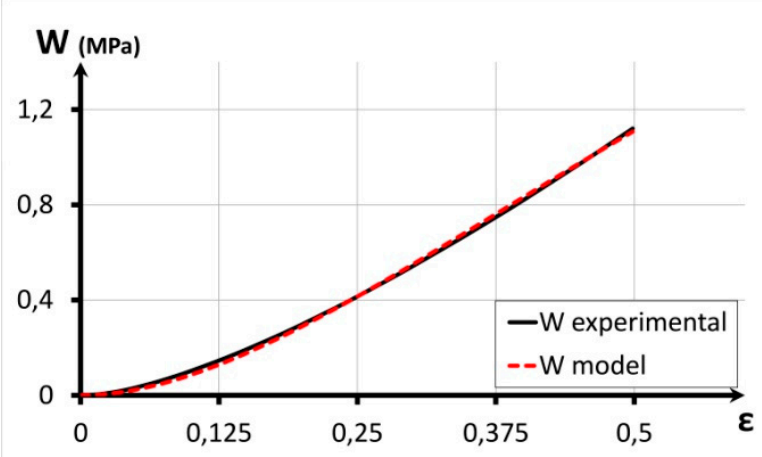

(a)

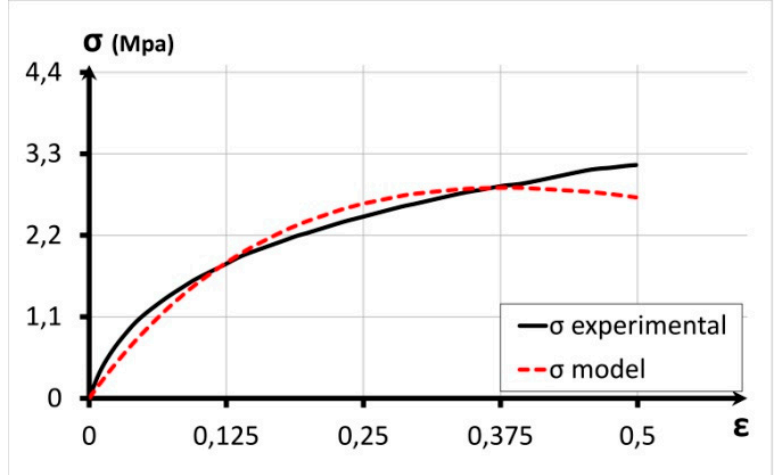

(b)

Figure 2. Mooney Rivlin model. (a) Strain-Strain energy density plot; (b) Strain-stress plot.

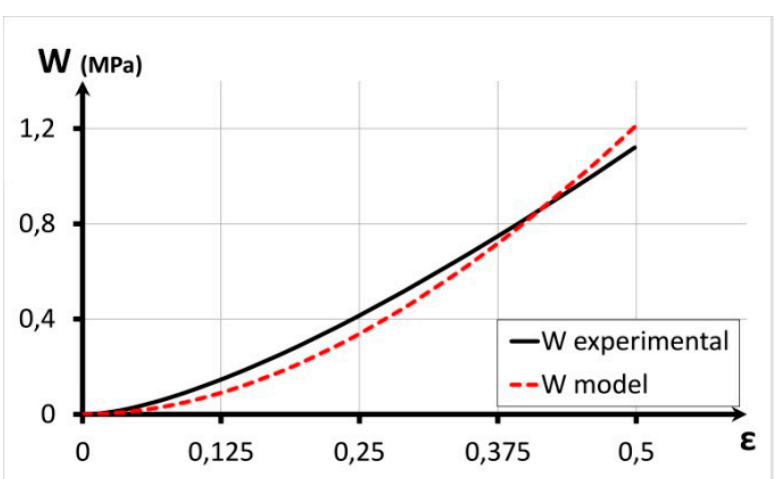

(a)

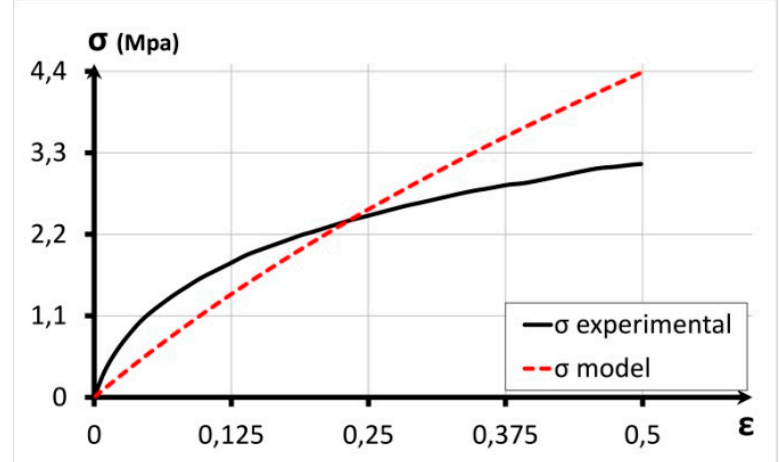

(b)

Figure 3. Neo Hookean model (a) Strain-Strain energy density plot; (b) Strain-stress plot. 


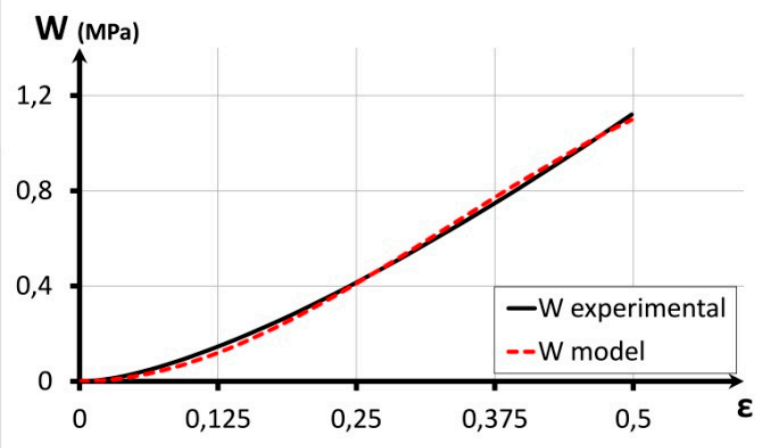

(a)

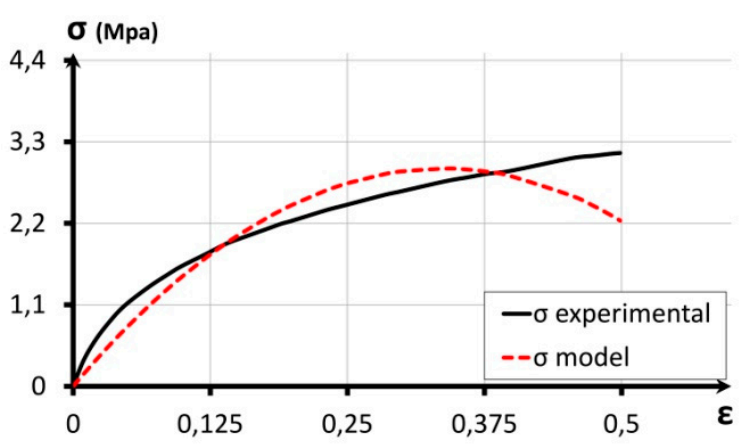

(b)

Figure 4. Ogden model. N=3 submodel. (a) Strain-Strain energy density plot; (b) Strain-stress plot.

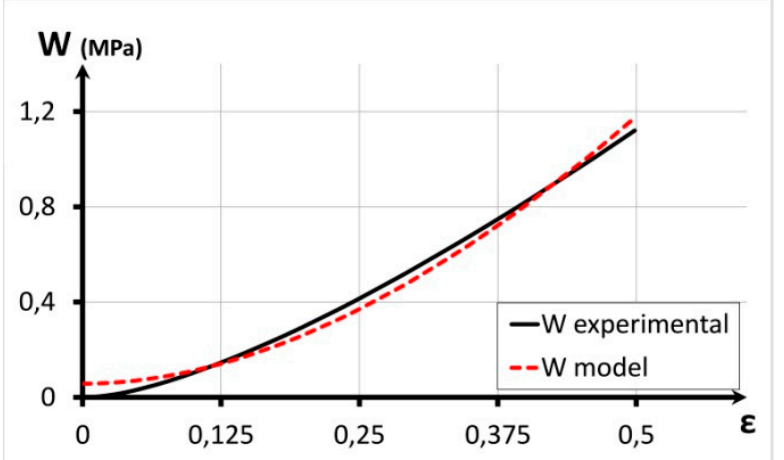

(a)

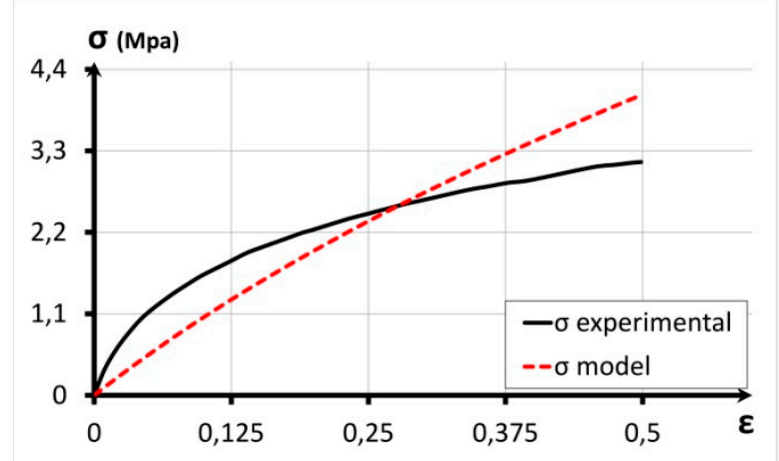

(b)

Figure 5. Gent-Thomas model. (a) Strain-Strain energy density plot; (b) Strain-stress

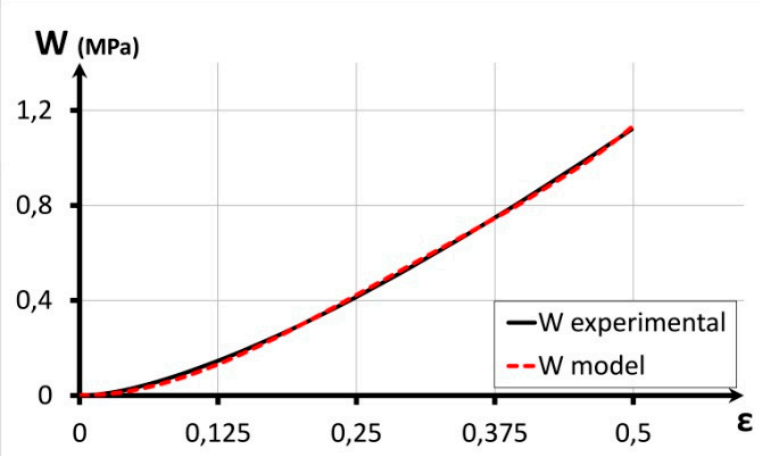

(a)

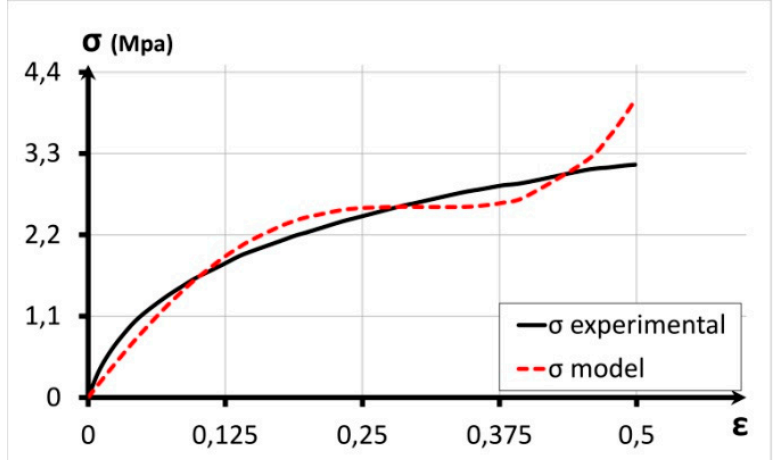

(b)

Figure 6. Yeoh model. N=3 submodel. (a) Strain-Strain energy density plot; (b) Strain-stress 


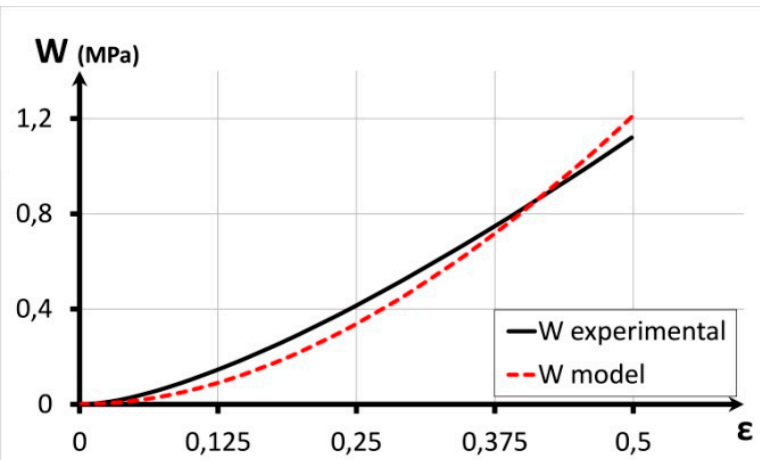

(a)

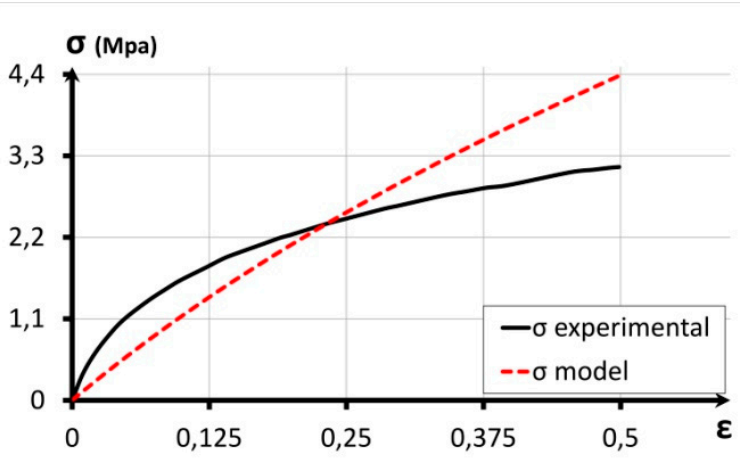

(b)

Figure 7. Arruda-Boyce model. N=1 submodel. (a) Strain-Strain energy density plot; (b) Strain-stress

The values of the material constants are shown for each of the different models studied in Tables 2 to 7 . Furthermore the value of $\mathrm{R}^{2}$ coefficient for strain energy density function and stress is shown.

Table 2. Mooney Rivlin model. 2 parameters submodel. Material parameters value and $\mathrm{R}^{2}$ coefficient for $\varepsilon-W$ and $\varepsilon-\sigma$ plots.

\begin{tabular}{|c|c|c|c|c|}
\hline Model & $\begin{array}{l}\text { Material } \\
\text { parameter }\end{array}$ & Value & $\mathbf{R}^{2}(\mathrm{~W})$ & $\mathbf{R}^{2}(\sigma)$ \\
\hline \multirow{2}{*}{$\begin{array}{c}\text { Mooney-Rivlin } \\
2 \text { par. }\end{array}$} & $\mathrm{C}_{10}$ & $-3,0670$ & \multirow{2}{*}{0,9994} & \multirow{2}{*}{0,9572} \\
\hline & $\mathrm{C}_{01}$ & 6,5250 & & \\
\hline
\end{tabular}

Table 3. Neo Hookean model. Material parameters value and $\mathrm{R}^{2}$ coefficient for $\varepsilon-W$ and $\varepsilon-\sigma$ plots.

\begin{tabular}{ccccc}
\hline Model & $\begin{array}{c}\text { Material } \\
\text { parameter }\end{array}$ & Value & $\mathbf{R}^{2}(\mathrm{~W})$ & $\mathbf{R}^{2}(\sigma)$ \\
\hline Neo Hookean & $\mathrm{C}_{10}$ & 2,0790 & 0,9893 & 0,9246 \\
\hline
\end{tabular}

Table 4. Ogden model. $\mathrm{N}=3$ submodel. Material parameters value and $\mathrm{R}^{2}$ coefficient for $\varepsilon-\mathrm{W}$ and $\varepsilon-\sigma$ plots.

\begin{tabular}{|c|c|c|c|c|}
\hline Model & $\begin{array}{c}\text { Material } \\
\text { parameter }\end{array}$ & Value & $\mathbf{R}^{2}(\mathrm{~W})$ & $\mathbf{R}^{2}(\sigma)$ \\
\hline \multirow{6}{*}{ Ogden $N=3$} & $\mu_{1}$ & $-3,0800$ & \multirow{6}{*}{0,9985} & \multirow{6}{*}{0,8704} \\
\hline & $\alpha_{1}$ & 4,7360 & & \\
\hline & $\mu_{2}$ & 4,0040 & & \\
\hline & $\alpha_{2}$ & 3,4520 & & \\
\hline & $\mu_{3}$ & 9,5680 & & \\
\hline & $\alpha_{3}$ & 1,3090 & & \\
\hline
\end{tabular}

Table 5. Gent-Thomas model. Material parameters value and $\mathrm{R}^{2}$ coefficient for $\varepsilon-\mathrm{W}$ and $\varepsilon-\sigma$ plots.

\begin{tabular}{ccccc}
\hline \multirow{2}{*}{ Model } & $\begin{array}{c}\text { Material } \\
\text { parameter }\end{array}$ & Value & $\mathbf{R}^{2}(\mathrm{~W})$ & $\mathbf{R}^{2}(\sigma)$ \\
\cline { 1 - 3 } Gent-Thomas & $\mathrm{C}_{1}$ & 1,9130 & & \\
\cline { 2 - 4 } & $\mathrm{C}_{2}$ & 0,0173 & & \\
\hline
\end{tabular}


Table 6. Yeoh model. $\mathrm{N}=3$ submodel. Material parameters value and $\mathrm{R}^{2}$ coefficient for $\varepsilon-\mathrm{W}$ and $\varepsilon-\sigma$ plots.

\begin{tabular}{ccccc}
\hline \multirow{2}{*}{ Model } & $\begin{array}{c}\text { Material } \\
\text { parameter }\end{array}$ & Value & $\mathbf{R}^{2}(\mathrm{~W})$ & $\mathbf{R}^{2}(\sigma)$ \\
\hline \multirow{3}{*}{ Yeoh N=3 } & $\mathrm{C}_{10}$ & 3,2000 & & \\
\cline { 2 - 3 } 0 & $\mathrm{C}_{20}$ & $-4,2630$ & 0,9996 & 0,9465 \\
\cline { 2 - 3 } & $\mathrm{C}_{30}$ & 3,6230 & & \\
\hline
\end{tabular}

Table 7. Arruda-Boyce model. $\mathrm{N}=1$ submodel. Material parameters value and $\mathrm{R}^{2}$ coefficient for $\varepsilon-\mathrm{W}$ and $\varepsilon-\sigma$ plots.

\begin{tabular}{ccccc}
\hline \multirow{2}{*}{ Model } & $\begin{array}{c}\text { Material } \\
\text { parameter }\end{array}$ & Value & $\mathbf{R}^{2}(\mathrm{~W})$ & $\mathbf{R}^{2}(\sigma)$ \\
\hline \multirow{2}{*}{$\begin{array}{c}\text { Arruda-Boyce } \\
\mathrm{N}=1\end{array}$} & $\mu$ & 1,5625 & & \\
\cline { 2 - 3 } & $\lambda_{\mathrm{L} 1}$ & 1,8000 & & \\
\cline { 2 - 3 } & $\mathrm{C}_{1}$ & 1,3306 & 0,9246 \\
\hline
\end{tabular}

\section{Discussion}

The results show that the mechanical behavior of Santoprene 101-73 can be adjusted precisely using different models of hyperelastic behavior, because all models provide a good correlation with the data supplied by the manufacturer.

Figure 17 shows that all hyperelastic models analyzed work relatively well because $\mathrm{R}^{2}>0.90$ in all cases. However, the Mooney-Rivlin hyperelastic model is the most capable model to reproduce the real behavior of the material. This model shows the highest correlation on strain energy density $(0,9994)$ and stresses $(0,9572)$.

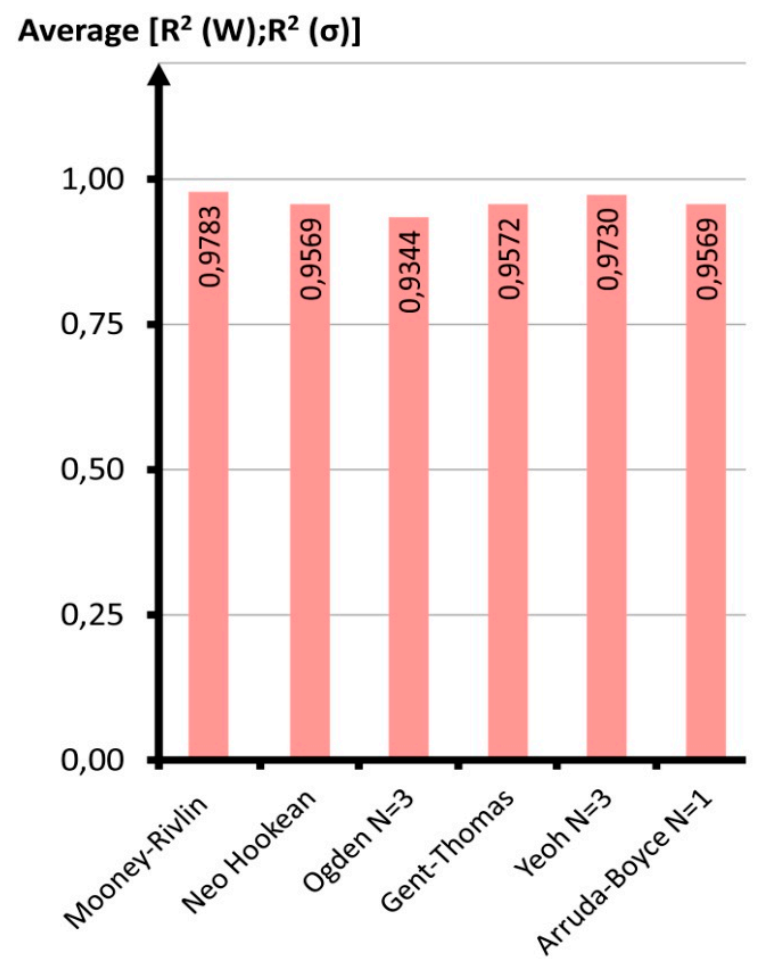

Figure 8. Average R2(W) and R2( $\sigma)$ values of hyperelastic models and submodels. 
Neo Hookean model is a particular case of Yeoh model with $\mathrm{N}=1$, with equivalent formulation, and therefore the results for both models are identical.

Attending to the stress results obtained, the importance of this kind of studies can be highlighted to perform mechanical simulations. Despite achieving $R^{2}$ values close to 1 in stress data results, the values of these stress in each model can be quite different from the actual values of the material obtained by experimental tests. These stress values will lead, in each section of the component, to resultant forces and moments quite different from the actual ones. It could, in some cases, cause problems in mounting and assembly phases of the designed parts.

\section{Conclusions}

The aim of this paper was to determine which is the best theoretical model for reproducing the mechanical behavior of Santoprene 101-73 material used in automotive industry. To reach it, 6 different hyperelastic models with their corresponding sub-models have been studied in order to obtain the material constants of each of them. The description of 15 hyperelastic formulations (models and submodels) have been made. An optimization least squares algorithm were used to fit the best values of material constants to each of them. In order to conclude which of them best represents the actual behavior of the material, the $\mathrm{R}^{2}$ correlation coefficient for two variables (strain energy density function and stress) has been used.

In view of the obtained results, it can be concluded that the Mooney-Rivlin model is the most accurate model to reproduce the mechanical behavior of Santoprene 101-73, being the 5 parameters submodel which provides the best results.

Acknowledgements: This research was partially supported by a Grant by S.A.U. Mann+Hummel Ibérica. This company has not had any influence on the study design, collection, analysis and interpretation of data, redaction of the manuscript and decision to submit this paper for publication.

Author contributions: L.G. and R.T. conceived the approach of this work, R.T. carried out all the calculations and performed the data verification. R. T. and E.I. performed the statistical analysis. L.G. coordinated the work. All authors participated in the drawing up of the manuscript, and read and approved the final manuscript.

Competing interests: The authors declare that there is no conflict of interest.

\section{References}

1. Štrumberger, N., Gospočić, A., \& Bartulić, Č. (2012). Polymeric Materials in Automobiles. PROMET-Traffic\&Transportation, 17(3), 149-160.

2. P. Consulting, Global elastomeric polyolefins markets, technologies trends (2014) 2014-2020.

3. Kutz, M. (Ed.). (2011). Applied plastics engineering handbook: processing and materials. William Andrew.

4. Robert A.. Malloy. (1994). Plastic part design for injection molding: an introduction. Hanser Publishers.

5. Drobny, J. G. (2014). Handbook of thermoplastic elastomers. Elsevier.

6. Dufton, P. W. (2001). Thermoplastic Elastomers Market. iSmithers Rapra Publishing.

7. D. J. Charlton, J. Yang, K. K. Teh, A Review of Methods to Characterize Rubber Elastic Behavior for Use in Finite Element Analysis, Rubber Chemistry and Technology (3) 481-503.

8. E. Mobil, Santoprene 101-73 Thermoplastic Vulcanizate.

9. M Mooney. A theory of large elastic deformation. Journal of applied physics, 11(9):582-592, 1940.

10. Ronald S Rivlin and DW Saunders. Large elastic deformations of isotropic materials. vii. experiments on the deformation of rubber. Philosophical Transactions of the Royal Society of London A: Mathematical, Physical and Engineering Sciences, 243(865):251-288, 1951.

11. LRG Treloar. The elasticity of a network of long-chain molecules. i. Transactions of the Faraday Society, 39:36-41, 1943. 
12. RW Ogden. Large deformation isotropic elasticity-on the correlation of theory and experiment for incompressible rubberlike solids. In Proceedings of the Royal Society of London A: Mathematical, Physical and Engineering Sciences, volume 326, pages 565-584. The Royal Society, 1972.

13. AN Gent and AG Thomas. Forms for the stored (strain) energy function for vulcanized rubber. Journal of Polymer Science, 28(118):625-628, 1958.

14. $\mathrm{OH}$ Yeoh. Characterization of elastic properties of carbon-black-filled rubber vulcanizates. Rubber chemistry and technology, 63(5):792-805, 1990.

15. Ellen M Arruda and Mary C Boyce. A three-dimensional constitutive model for the large stretch behavior of rubber elastic materials. Journal of the Mechanics and Physics of Solids, 41(2):389-412, 1993.

(C) 2016 by the authors; licensee MDPI, Basel, Switzerland. This article is an open access article distributed under the terms and conditions of the Creative Commons by Attribution (CC-BY) license (http://creativecommons.org/licenses/by/4.0/). 